$11-12-1996$

\title{
Potentials with Two Shifted Sets of Equally Spaced Eigenvalues and Their Calogero Spectrum
}

Asim Gangopadhyaya

Loyola University Chicago, agangop@luc.edu

Uday P. Sukhatme

University of Illinois at Chicago, sukhatme@uic.edu

Follow this and additional works at: https://ecommons.luc.edu/physics_facpubs

Part of the Quantum Physics Commons

\section{Author Manuscript}

This is a pre-publication author manuscript of the final, published article.

\section{Recommended Citation}

Gangopadhyaya, A and U Sukhatme. "Potentials with Two Shifted Sets of Equally Spaced Eigenvalues and Their Calogero Spectrum." http://arxiv.org/pdf/hep-th/9611086.pdf

This Article is brought to you for free and open access by the Faculty Publications and Other Works by Department at Loyola eCommons. It has been accepted for inclusion in Physics: Faculty Publications and Other Works by an authorized administrator of Loyola eCommons. For more information, please contact ecommons@luc.edu. cc) (i) $\Theta$

This work is licensed under a Creative Commons Attribution-Noncommercial-No Derivative Works 3.0 License. (C) 1996 The Authors. 


\title{
Potentials with Two Shifted Sets of Equally Spaced Eigenvalues and Their Calogero Spectrum
}

\author{
Asim Gangopadhyaya ${ }^{a}$, and Uday P. Sukhatme ${ }^{b \text {, }}$
}

a) Department of Physics, Loyola University Chicago, Chicago, Illinois 60626

b) Department of Physics (m/c 273), University of Illinois at Chicago, 845 W. Taylor Street, Chicago, Illinois 60607-7059

\begin{abstract}
Motivated by the concept of shape invariance in supersymmetric quantum mechanics, we obtain potentials whose spectrum consists of two shifted sets of equally spaced energy levels. These potentials are similar to the Calogero-Sutherland model except the singular term $\alpha x^{-2}$ always falls in the transition region $-\frac{1}{4}<\alpha<\frac{3}{4}$ and there is a $\delta$-function singularity at $x=0$.
\end{abstract}

\footnotetext{
${ }^{1}$ asim@uicws.phy.uic.edu, agangop@orion.it.luc.edu FAX: (773) 508-3534

${ }^{2}$ sukhatme@uic.edu FAX: (312) 996-9016
} 
It is well-known that a set of equally spaced energy eigenvalues corresponds to a particle moving in a harmonic oscillator potential. More specifically, in units of $\hbar=2 m=1$, the set of energy levels

$$
E_{n}=n \omega \quad(n=0,1,2, \ldots)
$$

corresponds to the one dimensional potential

$$
V(x)=\frac{1}{4} \omega^{2} x^{2}-\frac{1}{2} \omega \quad(-\infty<x<\infty) .
$$

However, if the particle is not allowed to move along the entire $x$-axis and is constrained to remain on the half-axis, a simple potential with eigenvalues given by eq. (1) is

$$
V(r)=\frac{1}{16} \omega^{2} r^{2}+\frac{l(l+1)}{r^{2}}-\frac{\omega}{2}\left(l+\frac{3}{2}\right) \quad\left(0<r<\infty ; \quad l \geq-\frac{1}{2}\right) .
$$

These potentials are not unique. It is well-known that they can be systematically deformed to produce multi-parameter isospectral families either via inverse scattering methods [1] or using supersymmetric quantum mechanics techniques [2].

Now, instead of a single set of equally spaced eigenvalues, let us make an innocuous extension and ask for potentials which possess two shifted sets of equally spaced eigenvalues. The energy levels are given by $n\left(\omega_{0}+\omega_{1}\right)$ and $n\left(\omega_{0}+\omega_{1}\right)+\omega_{0}$ with $n=0,1,2, \cdots$. Clearly, there are two sets of energy levels with equal spacing $\left(\omega_{0}+\omega_{1}\right)$, with one set displaced by $\omega_{0}$ with respect to the other set [see Fig. 1]. To our knowledge, potentials which possess the spectrum of Fig. 1 have not been discussed in the literature. We will obtain simple, explicit potentials using an approach motivated by recent developments [3, 㐰 in obtaining new shape invariant potentials [5] in supersymmetric quantum mechanics [2, 6]. These advances have been made using novel choices of the function $f$ appearing in the change of parameters $a_{1}=f\left(a_{0}\right)$ in the shape invariance condition. In this paper, we use a transformation such that $f^{2}\left(a_{0}\right)=a_{0}$. The resulting shape invariant potentials are found to be singular but analytically solvable and will have a spectrum consisting of two shifted sets of equally spaced energy levels. Other potentials with the same spectrum can be readily obtained by making isospectral deformations [2, 7], but this aspect will not be further developed here. We discuss applications to multiparticle problems [8, 9]. In particular, we find that there exist new eigenstates interlaced between the usual equally spaced Calogero spectrum of eigenvalues. These new states are a direct consequence of the fact that potentials with a 
$\alpha x^{-2}$ singularity with strength $\alpha$ in the transition region $-\frac{1}{4}<\alpha<\frac{3}{4}$, possess additional bound states with singular but normalizable wave functions.

\section{Shape Invariant Potentials:}

Let us recall that supersymmetric partner potentials $V_{ \pm}\left(x, a_{0}\right)$ are related to the superpotential $W\left(x, a_{0}\right)$ by

$$
V_{ \pm}\left(x, a_{0}\right)=W^{2}\left(x, a_{0}\right) \pm W^{\prime}\left(x, a_{0}\right)
$$

where $a_{0}$ is a set of parameters. These partner potentials are shape invariant if they both have the same $x$-dependence upto a change of parameters $a_{1}=f\left(a_{0}\right)$ and an additive constant $R\left(a_{0}\right)$. The shape invariance condition is

$$
V_{+}\left(x, a_{0}\right)=V_{-}\left(x, a_{1}\right)+R\left(a_{0}\right)
$$

or equivalently

$$
W^{2}\left(x, a_{0}\right)+W^{\prime}\left(x, a_{0}\right)=W^{2}\left(x, a_{1}\right)-W^{\prime}\left(x, a_{1}\right)+R\left(a_{0}\right)
$$

The property of shape invariance permits an immediate analytic determination of energy eigenvalues [5], eigenfunctions [10] and scattering matrices [11]. For unbroken supersymmetry, the eigenstates of the potential $V_{-}(x)$ are:

$$
\begin{gathered}
E_{0}^{(-)}=0, E_{n}^{(-)}=\sum_{k=0}^{n-1} R\left(a_{k}\right) \\
\psi_{0}^{(-)} \propto e^{-\int_{x_{0}}^{x} W\left(y, a_{0}\right) d y}, \psi_{n}^{(-)}\left(x, a_{0}\right)=\left[-\frac{d}{d x}+W\left(x, a_{0}\right)\right] \psi_{n-1}^{(-)}\left(x, a_{1}\right) \quad(n=1,2,3, \ldots) .
\end{gathered}
$$

Note the existence of a zero energy ground state, characteristic of unbroken supersymmetry.

\section{Change of Parameters:}

Until 1992, the only known solutions of the shape invariance condition corresponded to a translational change of parameters

$$
a_{1}=f\left(a_{0}\right)=a_{0}+\beta, a_{n}=f^{n}\left(a_{0}\right)=a_{0}+n \beta .
$$

Subsequently, the scaling change of parameters

$$
a_{1}=f\left(a_{0}\right)=q a_{0}, a_{n}=f^{n}\left(a_{0}\right)=q^{n} a_{0}
$$


was studied and shown to produce new classes of shape invariant potentials [3, 迎, which included self-similar potentials [12] as a special case. The above mentioned change of parameters are shown in Fig. 2. In general, the changes are monotonic, and the parameters never acquire the same values again. The only exceptions are the special cases $\beta=0$ and $q=1$, both of which have $f\left(a_{0}\right)=a_{0}$ and hence $a_{n}=a_{n-1}=\cdots=a_{1}=a_{0}$. For this situation,

the energy levels are equally spaced since $E_{n}^{(-)}-E_{n-1}^{(-)}=R\left(a_{0}\right)$. If one denotes the constant value of $R\left(a_{0}\right)$ by $\omega$, then the shape invariance condition [eq. (6)] reads $W^{\prime}\left(x, a_{0}\right)=\omega / 2$. This gives $W\left(x, a_{0}\right)=\omega x / 2$ and the standard harmonic oscillator potential $V_{-}(x)$ of eq. (2).

Let us now consider a new change of parameters defined by $f^{2}\left(a_{0}\right)=a_{0}$. Consequently,

$$
a_{0}=a_{2}=a_{4}=\cdots \quad, \quad f\left(a_{0}\right)=a_{1}=a_{3}=a_{5}=\cdots \quad .
$$

There are many functions which meet the above requirement. One such function is $f\left(a_{0}\right)=$ $C / a_{0}$, where $C$ is any real constant. Another choice is $f\left(a_{0}\right)=\frac{1}{2 C} \log \left[\operatorname{coth}\left(C a_{0}\right)\right]$. The energy levels will be spaced alternately by $R\left(a_{0}\right) \equiv \omega_{0}$ and $R\left(a_{1}\right) \equiv \omega_{1}$. This spectrum corresponds to two shifted sets of equally spaced eigenvalues.

\section{Single Particle Potentials:}

To explicitly compute the potentials associated with two shifted sets of equally spaced eigenvalues, one needs to solve the shape invariance conditions:

$$
\begin{aligned}
& W^{2}\left(x, a_{0}\right)+W^{\prime}\left(x, a_{0}\right)=W^{2}\left(x, a_{1}\right)-W^{\prime}\left(x, a_{1}\right)+\omega_{0}, \\
& W^{2}\left(x, a_{1}\right)+W^{\prime}\left(x, a_{1}\right)=W^{2}\left(x, a_{0}\right)-W^{\prime}\left(x, a_{0}\right)+\omega_{1} .
\end{aligned}
$$

The solution is readily obtained by straightforward manipulations. The superpotential $W\left(x, a_{0}\right)$ is given by

$$
W\left(x, a_{0}\right)=\frac{\left(\omega_{0}+\omega_{1}\right) x}{4}+\frac{\left(\omega_{0}-\omega_{1}\right)}{2 x\left(\omega_{0}+\omega_{1}\right)}
$$

and the corresponding potential $V_{-}(x)$ is

$$
V_{-}\left(x, a_{0}\right)=\frac{\left(\omega_{0}-\omega_{1}\right)\left(3 \omega_{0}+\omega_{1}\right)}{4\left(\omega_{0}+\omega_{1}\right)^{2} x^{2}}-\frac{\omega_{1}}{2}+\frac{\left(\omega_{0}+\omega_{1}\right)^{2} x^{2}}{16} .
$$

The above results are correct everywhere except at the point $x=0$, where the superpotential $W\left(x, a_{0}\right)$ has an infinite discontinuity. Such a discontinuity is not acceptable, since 
while writing eqs. (44) to (8), we have implicitly assumed the existence of a continuous superpotential with a well-defined derivative giving rise to unbroken supersymmetry and a zero energy ground state. To study the problem further, consider a regularised, continuous superpotential $\tilde{W}\left(x, a_{0}, \epsilon\right)$ which reduces to $W\left(x, a_{0}\right)$ in the limit $\epsilon \rightarrow 0$. One such choice is

$$
\tilde{W}\left(x, a_{0}, \epsilon\right)=W\left(x, a_{0}\right) f(x, \epsilon)
$$

where

$$
f(x, \epsilon)=\tanh ^{2} \frac{x}{\epsilon}
$$

The moderating factor $f$ provides a smooth interpolation through the discontinuity, since it is unity everywhere except in a small region of order $\epsilon$ around $x=0$. In this region, $\tilde{W}\left(x, a_{0}, \epsilon\right)$ is linear with a slope $\frac{\left(\omega_{0}-\omega_{1}\right)}{2 \epsilon^{2}\left(\omega_{0}+\omega_{1}\right)}$ which becomes larger as $\epsilon$ gets smaller. The potential $\tilde{V}_{-}\left(x, a_{0}, \epsilon\right)$ corresponding to the superpotential $\tilde{W}\left(x, a_{0}, \epsilon\right)$ is

$$
\tilde{V}_{-}\left(x, a_{0}, \epsilon\right)=\tilde{W}^{2}\left(x, a_{0}, \epsilon\right)-\tilde{W}^{\prime}\left(x, a_{0}, \epsilon\right)
$$

In the limit $\epsilon \rightarrow 0, \tilde{V}_{-}\left(x, a_{0}, \epsilon\right)$ reduces to

$$
\tilde{V}_{-}\left(x, a_{0}\right)=V_{-}\left(x, a_{0}\right)-4 W\left(x, a_{0}\right)[2 \theta(x)-1] \delta(x),
$$

where $\theta(x)$ is the unit step function and we have used $\lim _{\epsilon \rightarrow 0} \frac{1}{2 \epsilon} \operatorname{sech}^{2} \frac{x}{\epsilon}=\delta(x), \lim _{\epsilon \rightarrow 0} \tanh \frac{x}{\epsilon}=$ $[2 \theta(x)-1]$. Thus we see that the potential $\tilde{V}_{-}\left(x, a_{0}\right)$ has an an additional singularity at the origin over $V_{-}\left(x, a_{0}\right)$ given by $\Omega(x) \equiv-\frac{2\left(\omega_{0}-\omega_{1}\right)}{x\left(\omega_{0}+\omega_{1}\right)}[2 \theta(x)-1] \delta(x)$. Under scaling this singularity transforms as $\Omega(\lambda x)=\frac{1}{\lambda^{2}} \Omega(x) \quad(\lambda \geq 0)$. The sign of this singularity depends upon the values of $\omega_{0}$ and $\omega_{1}$. It is best to consider the two cases $\omega_{0}>\omega_{1}$ and $\omega_{0}<\omega_{1}$ separately.

Case 1: $\omega_{0}>\omega_{1}$. An example of a superpotential for this case is shown in Fig. 3(a). The corresponding potential is drawn in Fig. 1(a), along with a few low lying energy levels. Note the sharp attractive shape of the potential near $x=0$. In the limit $\epsilon \rightarrow 0$, this attractive $\delta$-function singularity at the origin is instrumental in producing a bound state at $E_{0}=0$.

Case 2: $\omega_{0}<\omega_{1}$. An example of a superpotential for this case is shown in Fig. 3(b). Again, proceeding as in case 1 , one gets a repulsive singularity in the potential at the origin. This is shown in Fig. 1(b).

Naively, in the limit $\epsilon \rightarrow 0$, the potential of eq. (10) appears identical to a three dimensional oscillator with a frequency $\omega \equiv \frac{\left(\omega_{0}+\omega_{1}\right)}{2}$ and angular momentum $l$ given by 
$l(l+1)=\frac{\left(\omega_{0}-\omega_{1}\right)\left(3 \omega_{0}+\omega_{1}\right)}{4\left(\omega_{0}+\omega_{1}\right)^{2}}$. However, there are some subtle but important differences. It is defined over the entire real axis $(-\infty<x<\infty)$ and not just the half line, and a very interesting feature of this potential is the inevitable "softness" of the inverse square term. For all values of parameters $\omega_{0}$ and $\omega_{1}$, the coefficient of the inverse square term is bounded between $-\frac{1}{4}$ and $\frac{3}{4}$; thus the potential falls exactly in what is known as the transition region 13, 14, 15]. More specifically, for case $1\left(\omega_{0}>\omega_{1}\right)$, one has $0<l(l+1)<\frac{3}{4}$ and for case $2\left(\omega_{0}<\omega_{1}\right)$ one has $-\frac{1}{4}<l(l+1)<0$. The important special case of the one dimensional harmonic oscillator has $\omega_{0}=\omega_{1}$ : it corresponds to $l(l+1)=0$ and no $x^{-2}$ singularity. For transition potentials, both solutions of the Schrödinger equation are square integrable at the origin. If one seeks eigenstates in a semi-infinite domain $(0 \leq x<\infty)$, one just keeps the less "singular" solution of the two 13, 14, 15. However, for a fully infinite domain $(-\infty<x<\infty)$ with a soft singularity at the origin, both are acceptable square integrable solutions of the Schrödinger equation, and must be retained to form a complete set. Single particle eigenstates for the potential $\tilde{V}_{-}\left(x, a_{0}\right)=V_{-}\left(x, a_{0}\right)+\Omega(x)$, where $\Omega(x)$ represents the point singularity at the origin, are readily obtained from eq. (7). The lowest four are:

$$
\begin{aligned}
& E_{0}=0 ; \quad \psi_{0} \propto x^{-\frac{\omega_{0}-\omega_{1}}{2\left(\omega_{0}+\omega_{1}\right)}} e^{-\frac{1}{8}\left(\omega_{0}+\omega_{1}\right) x^{2}}, \\
& E_{1}=\omega_{0} ; \quad \psi_{1} \propto x^{1+\frac{\omega_{0}-\omega_{1}}{2\left(\omega_{0}+\omega_{1}\right)}} e^{-\frac{1}{8}\left(\omega_{0}+\omega_{1}\right) x^{2}}, \\
& E_{2}=\omega_{0}+\omega_{1} ; \quad \psi_{2} \propto\left(\frac{\omega_{0}-\omega_{1}}{\omega_{0}+\omega_{1}}-1+\frac{\omega_{0}+\omega_{1}}{2} x^{2}\right) x^{-\frac{\omega_{0}-\omega_{1}}{2\left(\omega_{0}+\omega_{1}\right)}} e^{-\frac{1}{8}\left(\omega_{0}+\omega_{1}\right) x^{2}} \\
& E_{3}=2 \omega_{0}+\omega_{1} ; \quad \psi_{3} \propto\left(-\frac{\omega_{0}-\omega_{1}}{\omega_{0}+\omega_{1}}-3+\frac{\omega_{0}+\omega_{1}}{2} x^{2}\right) x^{1+\frac{\omega_{0}-\omega_{1}}{2\left(\omega_{0}+\omega_{1}\right)}} e^{-\frac{1}{8}\left(\omega_{0}+\omega_{1}\right) x^{2}} .
\end{aligned}
$$

In Fig. 4, we have drawn the lowest few eigenfunctions for two choices of the parameters $\omega_{0}$ and $\omega_{1}$. Note that for $\omega_{0}>\omega_{1}$, the ground state eigenfunction diverges at the origin, whereas for $\omega_{0}<\omega_{1}$ it vanishes. For the intermediate situation $\omega_{0}=\omega_{1}$, the ground state is finite at the origin and is just the standard Gaussian solution of a one dimensional oscillator. In Fig. 5, we have plotted the five lowest energy eigenvalues $E_{0}$ to $E_{4}$ as a function of $\alpha \equiv\left(\omega_{0}-\omega_{1}\right)\left(3 \omega_{0}+\omega_{1}\right) / 4\left(\omega_{0}+\omega_{1}\right)^{2}$ holding $\omega \equiv\left(\omega_{0}+\omega_{1}\right) / 2$ fixed. $\alpha$ is the coefficient of the singular term in eq. (10). $2 \omega$ is the equal energy spacing in the two sets of energy levels. General expressions for these eigenfunctions and corresponding eigenenergies 
are given by

$$
\begin{array}{cc}
E_{2 n}=n\left(\omega_{0}+\omega_{1}\right), & \psi_{2 n} \propto x^{-\frac{d}{2}} e^{-\frac{1}{4} \omega x^{2}} L_{n}^{-\frac{d}{2}-\frac{1}{2}}\left[\frac{\omega x^{2}}{2}\right] \\
E_{2 n+1}=n\left(\omega_{0}+\omega_{1}\right)+\omega_{0}, & \psi_{2 n+1} \propto x^{1+\frac{d}{2}} e^{-\frac{1}{4} \omega x^{2}} L_{n}^{\frac{d}{2}+\frac{1}{2}}\left[\frac{\omega x^{2}}{2}\right]
\end{array}
$$

where $d \equiv \frac{\omega_{0}-\omega_{1}}{\left(\omega_{0}+\omega_{1}\right)}, \omega \equiv \frac{\left(\omega_{0}+\omega_{1}\right)}{2}$, and $L_{n}$ are the standard Laguerre polynomials.

\section{Two Particle Case:}

Consider the case of two particles interacting via the potential $\tilde{V}_{-}\left(\frac{x_{1}-x_{2}}{\sqrt{2}}, a_{0}\right)$ of eq. (14), consisting of the Calogero part $V_{-}\left(\frac{x_{1}-x_{2}}{\sqrt{2}}, a_{0}\right)$ and the additional $\delta$-function singularity. The Calogero part [eq. (10)] has been extensively discussed in the context of solvable many body problems in one dimension [8]. However, as we mentioned earlier, if the coefficient $\alpha$ of the singular term in eq. (10) is chosen to be between $-\frac{1}{4}$ and $\frac{3}{4}$, the "softness" of the singularity permits the penetration of particles through the barrier at the origin. For the two particle case this means that particles are able to interchange their positions by passing through each other. This leads to the appearance of additional eigenstates that are absent for a potential with a hard singularity $\left(\alpha>\frac{3}{4}\right)$, where only those wave functions that vanish at the origin are allowed. As a consequence of this "tunneling", eigenstates break into groups of two, with the splitting of the two states vanishing as the coefficient approaches $\frac{3}{4}$. However, in our case, for the potential $\tilde{V}_{-}\left(\frac{x_{1}-x_{2}}{\sqrt{2}}, a_{0}\right)$, particles also see the point singularity $\Omega\left(\frac{x_{1}-x_{2}}{\sqrt{2}}\right)$ as they pass through each other. The corresponding Schrödinger equation is

$$
\left\{-\frac{\partial^{2}}{\partial x_{1}^{2}}-\frac{\partial^{2}}{\partial x_{2}^{2}}+\frac{\omega^{2}}{8}\left(x_{1}-x_{2}\right)^{2}+\frac{2 \alpha}{\left(x_{1}-x_{2}\right)^{2}}+\Omega\left(\frac{x_{1}-x_{2}}{\sqrt{2}}\right)\right\} \psi\left(x_{1}, x_{2}\right)=E \psi\left(x_{1}, x_{2}\right) .
$$

Changing variables to center of mass coordinate $R=\frac{x_{1}+x_{2}}{2}$, and relative coordinate $x=$ $\frac{x_{1}-x_{2}}{\sqrt{2}}$, and then eliminating the motion of the center of mass, we arrive at

$$
\left\{-\frac{d^{2}}{d x^{2}}+\frac{1}{4} \omega^{2} x^{2}+\frac{\alpha}{x^{2}}+\Omega(x)\right\} \psi(x)=E \psi(x) .
$$

The domain of $x$ is the entire real line. Thus, as discussed earlier for the single particle case, both solutions of the Schrödinger equation are necessary to generate a complete set of eigenstates on the full line. Now with the identification $\omega_{0}=2 a \omega$ and $\omega_{1}=2(1-a) \omega$, 
where $a=\frac{1}{2} \sqrt{1+4 \alpha}$, eq. (18) reduces essentially to eq. (3). From eq. (16), the energy eigenvalues are given by

$$
E_{2 n}=2 n \omega, \quad E_{2 n+1}=2 n \omega+2 a \omega .
$$




\section{Three Particle Case:}

The Schrödinger equation for a three particle system that we consider here is:

$$
\left\{-\sum_{i=1}^{3} \frac{\partial^{2}}{\partial x_{i}^{2}}+\sum_{\substack{i, j=1 \\ i \neq j}}^{3}\left(\frac{\omega^{2}}{8}\left(x_{i}-x_{j}\right)^{2}+\frac{2 \alpha}{\left(x_{i}-x_{j}\right)^{2}}+\Omega\left(\frac{x_{i}-x_{j}}{\sqrt{2}}\right)\right)\right\} \psi E \psi,
$$

where the singular function $\Omega(x)$ is given by $\Omega(x)=-\frac{4 b[2 \theta(x)-1]}{x} \delta(x)$, where $b$ is defined by $\alpha=b(b+1)$. To avoid "falling to the center" [13], we shall confine our attention to $\alpha>-\frac{1}{4}$. Transforming to center of mass and relative coordinates

$$
R=\frac{x_{1}+x_{2}+x_{3}}{3}, x=\frac{x_{1}-x_{2}}{\sqrt{2}}, y=\frac{x_{1}+x_{2}-2 x_{3}}{\sqrt{6}},
$$

and then eliminating the center of mass variable, we get

$$
\begin{aligned}
\left\{-\frac{\partial^{2}}{\partial x^{2}}-\frac{\partial^{2}}{\partial y^{2}}+\frac{3}{8} \omega^{2}\left(x^{2}+y^{2}\right)\right. & +\alpha\left(\frac{1}{x^{2}}+\frac{4}{(\sqrt{3} y-x)^{2}}+\frac{4}{(\sqrt{3} y+x)^{2}}\right) \\
+ & \left.=(x)+\Omega\left(\frac{\sqrt{3} y-x}{2}\right)+\Omega\left(\frac{\sqrt{3} y+x}{2}\right)\right\} \psi
\end{aligned}
$$

Variables $x$ and $y$ span a two dimensional configurational plane. We parametrize this plane in polar coordinates $r=\sqrt{x^{2}+y^{2}}$ and $\phi=\tan ^{-1} \frac{x}{y}$. The range of these new variables are given by $0<r<\infty$ and $0 \leq \phi<2 \pi$. The Schrödinger equation in the variables $r$ and $\phi$ is

$$
\begin{gathered}
\left\{-\frac{\partial^{2}}{\partial r^{2}}-\frac{1}{r} \frac{\partial}{\partial r}+\frac{3}{8} \omega^{2} r^{2}+\frac{1}{r^{2}}\left\{-\frac{\partial^{2}}{\partial \phi^{2}}+\right.\right. \\
\left.\left.\sum_{n=0}^{2}\left(\frac{\alpha}{\sin ^{2}\left(\phi+\frac{2 n}{3} \pi\right)}+\Omega\left(\sin \left(\phi+\frac{2 n}{3} \pi\right)\right)\right)\right\}\right\} \psi=E \psi ;
\end{gathered}
$$

where we have used $\Omega\left(r \sin \left(\phi+\frac{2 n}{3} \pi\right)\right)=\frac{1}{r^{2}} \Omega\left(\sin \left(\phi+\frac{2 n}{3} \pi\right)\right)$. Owing to the zeros of the function $\sin \left(\phi+\frac{2 n}{3} \pi\right)$, the $\Omega$-singularities are at $\frac{k \pi}{3} \quad(k=0,1, \cdots, 5)$ and they can be written as $\sum_{k=0}^{5} \Omega\left(\phi-\frac{k \pi}{3}\right)$. Substituting $\psi(r, \phi)=R(r) f(\phi)$, and then using the usual procedure for separation of variables, we get:

$$
\left(-\frac{\partial^{2}}{\partial r^{2}}-\frac{1}{r} \frac{\partial}{\partial r}+\frac{3}{8} \omega^{2} r^{2}+\frac{b_{l}^{2}}{r^{2}}\right) R(r)=E R(r)
$$

and

$$
\begin{aligned}
\left\{-\frac{\partial^{2}}{\partial \phi^{2}}+\alpha\left(\frac{1}{\sin ^{2} \phi}+\right.\right. & \left.\frac{1}{\sin ^{2}\left(\phi+\frac{2}{3} \pi\right)}+\frac{1}{\sin ^{2}\left(\phi+\frac{4}{3} \pi\right)}\right) \\
& \left.+\sum_{n=0}^{n=2} \Omega\left(\sin \left(\phi+\frac{2 n}{3} \pi\right)\right)\right\} f_{l}(\phi)=b_{l}^{2} f_{l}(\phi) .
\end{aligned}
$$


The radial equation is same as that of a three dimensional oscillator. Its eigenfunctions and eigenvalues are given by

$$
R_{n l}(r)=r^{b_{l}} \exp \left[-\frac{1}{4} \sqrt{\frac{3}{2}} \omega r^{2}\right] L_{n}^{b_{l}}\left[\sqrt{\frac{3}{8}} \omega r^{2}\right], \quad E_{n}=\sqrt{\frac{3}{2}}\left(2 n+b_{l}+1\right), \omega
$$

where $L_{n}^{b}$ are Laguerre polynomials. To determine energy eigenvalues $E_{n}$, we still have to solve eq. (24). We first make a simplification using the identity [8]

$$
\left(\frac{1}{\sin ^{2} \phi}+\frac{1}{\sin ^{2}\left(\phi+\frac{2}{3} \pi\right)}+\frac{1}{\sin ^{2}\left(\phi+\frac{4}{3} \pi\right)}\right)=\frac{9}{\sin ^{2}(3 \phi)} .
$$

Thus eq. (24) now reduces to

$$
\left\{-\frac{\partial^{2}}{\partial \phi^{2}}+\frac{9 \alpha}{\sin ^{2} 3 \phi}+9 \sum_{n=0}^{n=5} \Omega(3 \phi-n \pi)\right\} f_{l}(\phi)=b_{l}^{2} f_{l}(\phi),
$$

where once again we have used the scaling property of the singular function $\Omega$. Since the effective potential of eq. (26) is periodic with a period $\pi / 3$, we only need to obtain a solution in the domain $0<\phi<\frac{\pi}{3}$. Using even and oddness of eigenfunctions, solutions can be extended beyond $\phi=\pi / 3$, as lucidly discussed in ref. [8]. Changing variables to $\xi=3 \phi$ yields

$$
\left\{-\frac{\partial^{2}}{\partial \xi^{2}}+\frac{\alpha}{\sin ^{2} \xi}+\Omega(\xi)+\Omega(\xi-\pi)\right\} f_{l}(\xi)=B_{l}^{2} f_{l}(\xi), \text { where } B_{l}=\frac{b_{l}}{3} .
$$

The new domain is $0 \leq \xi<\pi$. The major role played by the $\Omega$-singularity at the origin is to properly describe the discontinuity suffered by derivatives of the all even-states at $\xi=0$ and $\xi=\pi$. Now we perform another change of the independent variable from $\xi$ to $z=\cos ^{2} \frac{\xi}{2}$, and define $f_{l}(z)=[z(1-z)]^{\delta} h(z)$, where $\delta$ will be chosen judiciously to remove the singularity in eq. (27). For $\delta=\frac{1+\sqrt{1+4 \alpha}}{4}$, we get

$$
z(1-z) \frac{d^{2} h}{d z^{2}}+\left[2 \delta+\frac{1}{2}-(1+4 \delta) z\right] \frac{d h}{d z}-\left[4 \delta^{2}-B_{l}^{2}\right] h(z)=0 .
$$

This has the form of a hypergeometric equation and the general solution is

$$
\begin{aligned}
f_{l}(z) & =c_{1} z^{\delta}(1-z)^{\delta} F\left[2 \delta+B_{l}, 2 \delta-B_{l}, \frac{1}{2}+2 \delta, z\right] \\
& +c_{2} z^{\frac{1}{2}-\delta}(1-z)^{\delta} F\left[\frac{1}{2}+B_{l}, \frac{1}{2}-B_{l}, \frac{3}{2}-2 \delta, z\right] .
\end{aligned}
$$

Usually one of these solutions has an unacceptable singularity near $z \approx 0$, and the other solution is retained. However, for softly singular cases $\left(-\frac{1}{4}<\alpha<\frac{3}{4}\right)$ both solutions are 
normalizable, and necessary to generate a complete set of eigenfunctions. We would also like to emphasize that for the $\alpha \rightarrow 0$ limit, there is a smooth and continuous transition to one dimensional harmonic oscillator solutions (even and odd). The general solutions of eq. (26) in the domain $0<\phi<\pi / 3$ are given by

$$
\begin{aligned}
& f_{2 l}(\phi) \sim\left(\cos \frac{3 \phi}{2}\right)^{\frac{1}{2}-a}\left(\sin \frac{3 \phi}{2}\right)^{\frac{1}{2}+a} F\left[-2 l, 2 l+1,1-a ; \cos ^{2} \frac{3 \phi}{2}\right], \quad b_{2 l}=3\left(2 l+\frac{1}{2}\right), \\
& f_{2 l+1}(\phi) \sim(\sin 3 \phi)^{\frac{1}{2}+a} F\left[-2 l-1,2 l+2+2 a, 1+a ; \cos ^{2} \frac{3 \phi}{2}\right], \quad b_{2 l+1}=3\left(2 l+a+\frac{3}{2}\right),
\end{aligned}
$$

where $a=\frac{\sqrt{1+4 \alpha}}{2}$. The states $f_{2 l}(\phi)\left(f_{2 l+1}(\phi)\right)$ are symmetric (anti-symmetric) about the point $\phi=\frac{\pi}{6}$. To determine wave functions for any value of $\phi$, one uses $f_{l}(\phi)=f_{l}\left(\phi-\frac{m \pi}{3}\right)$, where $m$ is an appropriately chosen integer such that $0 \leq \phi-\frac{m \pi}{3} \leq \frac{\pi}{3}$. The eigenvalues are given by $E_{n, 2 l}=\sqrt{\frac{3}{2}}\left(2 n+6 l+\frac{5}{2}\right) \omega$ and $E_{n, 2 l+1}=\sqrt{\frac{3}{2}}\left(2 n+6 l+3 a+\frac{11}{2}\right) \omega$.

\section{Discussion and Comments:}

In this paper, we have shown that with a change of parameters of the type $a_{2} \equiv f^{2}\left(a_{0}\right)=$ $a_{0}$, one gets interesting shape invariant potentials with two shifted sets of equally spaced eigenvalues. These potentials are similar to the ones discussed by Calogero $[8]$. The main differences are: (a) the coefficient $\alpha$ of the inverse square term is always of the "soft" type i.e. $-\frac{1}{4}<\alpha<\frac{3}{4}$ and (b) there is an additional $\delta$-function singularity at the origin (in the multi-particle case this is a two-body contact interaction). For multi-particle systems that interact via two-body potentials of the above type, the spectrum includes the entire set eigenvalues of the Calogero potential as well as another set of equally spaced eigenvalues which are slightly shifted from Calogero set.

Can the same technique be extended, for example, to $a_{3} \equiv f^{3}\left(a_{0}\right)=a_{0}$, thus giving three shifted sets of equally spaced eigenvalues? This does not seem to be the case. Not only are there no simple real solutions to the constraint $f^{3}\left(a_{0}\right)=a_{0}$, but the shape invariance condition equations analogous to eq. (8) are not easily solvable. Other choices for the change of parameters $a_{1}=f\left(a_{0}\right)$ are currently being investigated.

A.G. acknowledges the hospitality of the UIC Department of Physics where part of this work was done. We also would like to thank R. Dutt and A. Khare for many valuable discussions and comments. Partial financial support from the U.S. Department of Energy is gratefully acknowledged. 


\section{References}

[1] K. Chadan and P. C. Sabatier, Inverse Problems in Quantum Scattering Theory, Springer Verlag (1977).

[2] For a recent review of supersymmetric quantum mechanics and additional references see F. Cooper, A. Khare and U. Sukhatme, Phys. Rep. 251 (1995) 267.

[3] A. Khare and U. Sukhatme, Jour. Phys. A26 (1993) L901.

[4] D. Barclay, R. Dutt, A. Gangopadhyaya, A. Khare, A. Pagnamenta and U. Sukhatme, Phys. Rev. A48 (1993) 2786.

[5] L. Gendenshtein, JETP Letters 38 (1983) 356.

[6] E. Witten, Nucl. Phys. B188 (1981) 513.

[7] A. Khare and U. Sukhatme, Jour. Phys. A22 (1989) 2847; W.-Y. Keung, U. Sukhatme, Q. M. Wang and T. Imbo, Jour. Phys. A22 (1989) L987: C. V. Sukumar, Jour. Phys. A19 (1986) 2297 and A20 (1987) 2461.

[8] F. Calogero, Jour. Math. Phys. 10 (1969) 2191, F. Calogero, Jour. Math. Phys. 12 (1971) 419.

[9] B. Sutherland, Jour. Math. Phys. 12 (1971) 246.

[10] R. Dutt, A. Khare and U. Sukhatme, Phys. Lett. 181B (1986) 295; J. Dabrowska, A. Khare and U. Sukhatme, Jour. Phys. A21 (1988) L195.

[11] A. Khare and U. Sukhatme, Jour. Phys. A21 (1988) L501.

[12] A. Shabat, Inverse Prob. 8 (1992) 303; V. Spiridonov, Phys. Rev. Lett. 69 (1992) 398.

[13] L. D. Landau and E. M. Lifshitz, Quantum Mechanics, Pergamon Press (1977).

[14] W. M. Frank, D. J. Land and R. M. Spector, Rev. Mod. Phys. 43 (1971) 36.

[15] A. Gangopadhyaya, P. K. Panigrahi and U. P. Sukhatme, J. Phys. A27 (1994) 4295. 


\section{Figure Captions}

Figure 1: The potentials $\tilde{V}_{-}\left(x, a_{0}, \epsilon\right)$ given by eq. (13) for the value $\epsilon=0.1$ and for two choices of parameters: (a) $\omega_{0}=1.7, \omega_{1}=0.3$; (b) $\omega_{0}=0.3, \omega_{1}=1.7$. The potentials correspond to the superpotentials of Fig. 1. The eigenvalue spectrum, consisting of two shifted sets of equally spaced energy levels, is given by eq. (16) and corresponds to the limit $\epsilon \rightarrow 0$.

Figure 2: Graphical representation of various modes of changing parameters $a_{n}=f^{n}\left(a_{0}\right)$ in the shape invariance condition. The variation of $a_{n}$ versus $n$ is shown by the symbol + for translations $a_{n}=a_{0}+n \beta$ and by the symbol $*$ for scaling $a_{n}=q^{n} a_{0}$. An example of the new change of parameters discussed in this paper is $f\left(a_{0}\right)=\frac{1}{2 C} \log \left[\operatorname{coth}\left(C a_{0}\right)\right]$, and the results are shown by the symbol o. The numerical choices for this figure are $a_{0}=0.8, \beta=0.1, q=0.9, C=0.5$.

Figure 3: The superpotentials $\tilde{W}\left(x, a_{0}, \epsilon\right)$ given by eq. (11). The curves are drawn for a small value $\epsilon=0.1$, in order to see the behavior near the origin. There are two choices of parameters: (a) $\omega_{0}=1.7, \omega_{1}=0.3$; (b) $\omega_{0}=0.3, \omega_{1}=1.7$.

Figure 4: The low lying eigenfunctions given by eq. (15) corresponding to the potentials of Fig. 1.

Figure 5: A plot of the five lowest energy eigenvalues $E_{0}$ to $E_{4}$ as a function of $\alpha \equiv$ $\left(\omega_{0}-\omega_{1}\right)\left(3 \omega_{0}+\omega_{1}\right) / 4\left(\omega_{0}+\omega_{1}\right)^{2}$ holding $\omega \equiv\left(\omega_{0}+\omega_{1}\right) / 2$ fixed. $\alpha$ is the coefficient of the singular term in eq. (10). $2 \omega$ is the equal energy spacing in the two sets of energy levels. The two sets are displaced by energy $\omega_{0}$. Regions shown by dashed lines correspond to singular but normalizable wave functions. 
This figure "fig1-1.png" is available in "png" format from: http://arxiv.org/ps/hep-th/9611086v1 
This figure "fig2-1.png" is available in "png" format from: http://arxiv.org/ps/hep-th/9611086v1 
This figure "fig1-2.png" is available in "png" format from: http://arxiv.org/ps/hep-th/9611086v1 
This figure "fig2-2.png" is available in "png" format from: http://arxiv.org/ps/hep-th/9611086v1 
This figure "fig1-3.png" is available in "png" format from: http://arxiv.org/ps/hep-th/9611086v1 